\title{
ANÁLISIS DE LAS ORIENTACIONES DE LOS ESTILOS DE INTERACCIÓN DEL TUTOR A DISTANCIA CON SUS ESTUDIANTES: APORTES PARA MEJORAR LA EFICACIA DEL PROCESO TUTORIAL
}

\section{Analysis of the orientations in the interaction styles of the online tutors and their students: contributions to promote the effectiveness of the tutorial process}

\author{
Dr. Néstor Fernández Sánchez ${ }^{1}$ \\ Universidad Nacional Autónoma de México
}

Recibido: $17-2-2015$

Aceptado: 10-6-2015

\section{RESUMEN}

La problemática que una universidad a distancia presentó con relación al incumplimiento de los estudiantes en la presentación de reportes de las actividades de aprendizaje motivó la realización de un estudio que analice el discurso producido en la interacción estudiantes- tutor. Se manejó la hipótesis de que los estilos de interacción de los tutores inciden en el desaliento de los estudiantes. Es un estudio exploratorio de corte longitudinal, que constituye la primera fase de un ejercicio más amplio a nivel comparativo con el cual se pretende identificar la orientación de los estilos de interacción de los tutores con sus estudiantes y su relación con la eficacia del proceso tutorial. Se contó con una muestra de 36 tutores activos y la compilación de los mensajes entre éstos y sus estudiantes, registrados en la plataforma educativa que soporta el desarrollo de los programas. El reporte describe la forma en que desde el análisis del discurso se fueron clasificando y depurando 2,081 registros de los discursos con el apoyo de OpenCode. En los resultados obtenidos se observa que los tutores ejercen una variedad de estilos de interacción, el $48 \%$ se orientaron hacia la realimentación del aprendizaje, el $8,48 \%$ confirman el incumplimiento de sus actividades y el $0,6 \%$ de mensajes se identificaron como expresiones con intención motivadora. El resto de los mensajes contenían argumentos relacionados con errores de ortografía $(3,6 \%)$, plagio $(5,2 \%)$, instrucciones no acatadas $(9,4 \%)$, errores en la redacción $(10,1 \%)$, falta de profundidad $(15,9 \%)$, confusión de contenidos o procedimientos $(17,8 \%)$ y carencia de elementos solicitados $(20,5 \%)$. Estos resultados servirán para la fase siguiente, centrada en la comparación de los estilos de atención del tutor y el desaliento de los estudiantes para evaluar la eficacia de los tutores en la forma de trabajo a distancia.

Palabras Clave: Tutor a distancia, educación a distancia, análisis del discurso, eficacia del proceso tutorial y estilos de interacción.

\section{Abstract}

Based on the problems that a distance university faced regarding with the students non-completion when handing in the reports of their learning activities, prompted us to conduct a study that analyses the discourse produced in the student-tutor interaction. The hypothesis states that the interaction styles of the tutors influence in the students discouragement. 
This is a longitudinal exploratory study that constitutes the first phase of a broader exercise at a comparative level which aims to identify the orientation of the interaction styles of the tutors and their students, and its relationship with the efecctiveness of the tutorial process. The sample was composed of 36 active tutors and the collecting of messages among them and their students, registered in the educational platform that supports the development of the programs. The report describes the way in which 2081 speech records were sorted and purged since the discourse analysis, supported by OpenCode. In view of the results, we can confirm that tutors have a variety of interaction styles, $48 \%$ were oriented towards learning feedback, the $8.48 \%$ confirm the noncompletion of their activities and the $0,6 \%$ of the messages were identified as expressions with motivating intention. The rest of the messages contained arguments related to misspellings (3.6\%), plagiarism (5.2\%), instructions not followed $(9.4 \%)$, mistakes in writing $(10.1 \%)$, lack of depth ( $15.9 \%)$, confusion of contents or procedures $(17.8 \%)$ and lack of requested items $(20.5 \%)$. The results will be used in the next phase, focused on the comparison of the tutor's styles of attention and the students' discouragement to evaluate the effectiveness of the tutors in distance learning. Keywords: online tutor, distance education, discourse analysis, tutorial effectiveness and interaction styles.

\section{INTRODUCCIÓN}

Hoy en día es indudable el impacto de las Tecnologías de la Información y la Comunicación (TIC) en las actividades cotidianas, sociales, profesionales y en la educación de casi todos los países. El uso de los recursos de Internet tiene beneficios innegables en la educación, de manera especial en la Educación a Distancia (EAD), donde es cada vez más evidente.

Quienes están inmersos en el campo de la educación saben y entienden que todo programa educativo requiere una planeación cuidadosa para propiciar el logro de los objetivos previstos. Esto tiene más sentido en la EAD, en la cual los procesos de aprendizaje-enseñanza se desarrollan sin la presencia "cara a cara" del docente. En la EAD es recomendable que el proceso de enseñanza centre la atención en el eduando y, por supuesto, en el logro de su aprendizaje significativo (Castañeda, 1993; Ausubel, 1978; Gallego y Martínez, 2003; Eusse, Fernandez, N. Análisis de las orientaciones de los estilos de interacción del tutor a distancia con sus estudiantes: aportes para mejorar la eficacia del proceso tutorial 2005). De hecho, cualquier modalidad que se pretenda instaurar en la educación debe ser eficaz y la Educación a Distancia ha demostrado dicho atributo (Aretio y Ruiz, 2010). Las opiniones en este tema exponen diversas aproximaciones teóricas, verbigracia, en el campo de la psicología del aprendizaje y los enfoques pedagógicos, que incluyen la posibilidad de valorar cómo se desarrollan los ambientes virtuales de aprendizaje (Moreno, 2003) y la comprensión de las características de las TIC y su potencial efecto sobre los procesos de construcción del conocimiento (Bustos y Coll, 2010; García, 2012); dentro del cual está inmersa la orientación de los estilos de interacción, la misma que debe llevar a mejorar la eficacia del proceso de tutoría.

El tema de la EAD puede verse desde varias perspectivas. Uno de los pioneros en escribir sobre ello es Lorenzo García Aretio, quien analizó diversas posturas teóricas y aportó, entre otras ideas, lo siguiente:

La enseñanza a distancia es un sistema tecnológico de comunicación bidireccional, que puede ser masivo, basado en la acción sistemática y conjunta de recursos didácticos y el apoyo de una organización y tutoría que, separados físicamente de los estudiantes, propician en estos un aprendizaje independiente (García, 1987 p. 9).

Dicha acción sistemática implica la práctica de la investigación permanente, con lo que se podrá coadyuvar a la prestación de un servicio efectivo que refleje los resultados esperados en los programas educativos. En otras palabras, se debe procurar la calidad de la educación a través del uso de una metodología pertinente, con estilos de in- 
Análisis de las orientaciones de los estilos de interacción del tutor a distancia con sus estudiantes: aportes para mejorar la eficacia del proceso tutorial

teracción pertinentes y adecuados en mejora del proceso de tutoria.

Si bien es cierto la EAD no es una opción nueva para apoyar a quienes desean estudiar y no tienen posibilidad de acudir a las aulas (Bosco y Barrón, 2008), la incorporación de las TIC ha hecho nueva cuenta del tema en los sistemas escolares. Estudiosos de este contexto, como Bates (1999), García $(2001,2007)$ y Crovi (2009), entre otros, coinciden en el concepto y aportes:

1. Ambos actores del proceso educativo, docente y estudiante, se encuentran separados en distancia y a veces en tiempo.

2. El uso de las TIC juega un papel de suma importancia para los fines educativos, toda vez que los reúne como pares o iguales en un ambiente virtual.

3. Es de suma importancia la comunicación que debe existir en ambos sentidos, tutor-alumno y viceversa para garantizar la calidad del proceso de aprendizaje-enseñanza.

Con base en los aportes de los autores mencionados, en este estudio se considera a la EAD como la modalidad en la que los actores del sistema educativo (institución, docente y alumnos) no interactúan de manera presencial, sino que lo hacen mediante las TIC y diversos recursos disponibles en la Web, contando con el apoyo de una diversidad de softwares o plataformas educativas auxiliados por recursos tecnológicos.

A diferencia de los sistemas tradicionales en los que la figura de un profesor se presenta a un grupo de estudiantes, en esta modalidad se asigna un acompañante: el tutor o asesor, facilitador o mentor, cuyo papel es personalizar el proceso educativo mediante el apoyo sistemático y cuidadosamente organizado.

\section{La eficacia del proceso tutorial}

Más allá de un análisis semántico o epistemológico de una definición de tutor, en los diversos aportes se concibe a la acción tutorial como el conjunto de tareas de quién interactúa con el educando -en este caso, a distancia-, respecto de los contenidos y procesos de aprendizaje. Como marco de refe- rencia, para soportar dicha afirmación se es preciso revisar las definiciones expresadas por diversos especialistas en la materia. García Aretio afirmó que "Se trata de un orientador del alumno que da respuesta por correo a las dudas presentadas por éste, devuelve los trabajos corregidos, anima al estudiante para que no abandone los estudios e incluso mantiene contactos presenciales con él...” (1999, p. 61). Ezequiel Ander-Egg (1977), en su diccionario define a la tutoría como la "acción de ayudar, guiar, aconsejar y orientar a los alumnos por parte de un profesor..."; es decir, el tutor es el guía, consejero u orientador del estudiante. En ambas definiciones se considera al tutor como la persona que ayuda al educando a lo largo del proceso de aprendizaje.

Si el tutor cumpliera con dichas características, se podría afirmar que cumple con su función, es decir, realiza una labor eficaz, logra en el estudiante el efecto previsto (Sander, 1990; Machado y Campos, 2013; Real Academia Española, 2015).

Cuando se habla de "animar al estudiante", rescátese el sentido afectivo, emocional y personal cuando de la mencionada función tutorial. Esta noción acerca a las tareas propias del tutor; algunas de corte técnico, como la de corregir trabajos y otras acciones orientadas al acompańamiento, como animar al estudiante a lo largo de todo su proceso de aprendizaje para que no se sienta solo y abandonado (Rodríguez, 2014).

En la definición de García Aretio se explicita la posibilidad de que el tutor evalúe los trabajos de los educandos y procure que los estudiantes no abandonen el programa. Como afirmó en su momento Cabero (2004), la función tutorial surge a reclamo del estudiante y el profesor, quien debe adoptar una posición más activa, interviniendo e indagando sobre situaciones problemáticas que vayan apareciendo a lo largo del proceso. Es decir, en la EAD se le asigna al tutor un papel más activo. Este no debe esperar a que el educando se le acerque, sino que buscará al estudiante durante todo el proceso educativo orientándole y motivándole para el logro de los objetivos del programa. Son parte de la funciones del tutor: estimular y orientar al alumno, facilitar situaciones de aprendizaje y ayudar a resolver los distintos ti- 
pos de dificultades que pudieran presentarse en su aprendizaje.

Con el apoyo de las TIC, el docente, ahora tutor, tiene la posibilidad de un acercamiento a sus estudiantes, gracias al apoyo de recursos de comunicación sincrónica o asincrónica; con ello se pretende garantizar la efectividad de la labor tutorial en el proceso de interactividad didáctica.

Un interesante aporte teórico sobre este tema se expuso en el documento Los tutores en la educación a distancia. Un aporte teórico, donde, entre otros puntos relevantes, se mencionan varias tareas del tutor a distancia (Pagano, 2008). El tutor tiene la tarea de lograr la confianza del alumno en el sistema de educación a distancia, orientándolo en la metodología que caracteriza a esta. Por ello debe conocer los fundamentos de la formación a distancia, las funciones que debe cumplir y las estrategias a emplear en la mediación pedagógica. El tutor debe realizar un monitoreo permanente de los progresos de sus estudiantes; enseñará, orientará, e integrará al alumno en el sistema por lo que debe poseer los siguientes atributos:

Empatía. Así logrará una cordialidad afectivo- social con sus alumnos, a pesar de la disociación del tiempo y el espacio y de la multiplicidad de estilos personales de estos.

Proacción. Ayudará a sortear los obstáculos y resistencias que se le presenten al estudiante en el contexto tecnológico, como en lo humano.

Anfitrión. Tendrá la afabilidad suficiente para introducir y mantener motivados a los alumnos en esta modalidad educativa.

Comunicación. Manejará todas las posibilidades y alternativas de comunicación en los distintos soportes tecnológicos, al mismo tiempo que será claro y evitará distorsionar la esencia de los mensajes. A su vez, será un mediador entre las múltiples comunicaciones generadas por la interacción entre los alumnos y él.

Didáctica. Seleccionará los contenidos y diseñará las actividades pertinentes para el logro de los objetivos propuestos, ajustándose al perfil del grupo de alumnos y las características individuales de sus integrantes.
Una aproximación similar a la de Pagano se halla en el documento La enseñanza a distancia y el rol del tutor virtual: una visión desde la Sociedad del Conocimiento (Fernández, Mireles y Aguilar, 2010). Como parte de las cualidades del tutor, se hace énfasis en la capacidad de cordialidad, la aceptación del otro (el estudiante), la empatía y la capacidad de escucha. También se ha enfatizado el papel del tutor a distancia en el tema de lo socio afectivo y la forma en que este interactúa con sus estudiantes, ya que la postura cognoscitiva subraya la importancia de la atención hacia el sujeto discente como persona. Las evidencias de dicha interacción se rescatan fácilmente desde los registros de la comunicación escrita entre ambos actores (Turizo, 2014).

Lo anteriormente comentado parece lógico y natural. Pero, pareciera que no es así, pues los sistemas a distancia pioneros incorporaron a docentes presenciales, convirtiéndoles en tutores, sin contar con un modelo sólido o comprobado acerca del compromiso y funciones de este actor. Con relación a este tema, en México existe una norma de competencia laboral para "Tutoría de cursos de formación en línea” (SEP, 2009). Es de suponerse que, según esta norma, toda persona interesada en el tema debe cubrir los requisitos establecidos para desempeñarse como tutor a distancia. Después de más de diez años de experiencia en la planeación, desarrollo y evaluación de este tipo de procesos educativos, el autor de este artículo ha llegado a la conclusión de que solo se someten a este tipo de evaluación quienes intentan incorporarse a organizaciones o instituciones privadas de corte empresarial; lo hacen con la finalidad de formar parte de los equipos de capacitación laboral. No ocurre así con quienes se encuentran o pretenden ser parte de los programas que se desarrollan en las instituciones educativas. 
Análisis de las orientaciones de los estilos de interacción del tutor a distancia con sus estudiantes: aportes para mejorar la eficacia del proceso tutorial

\section{La Educación a Distancia}

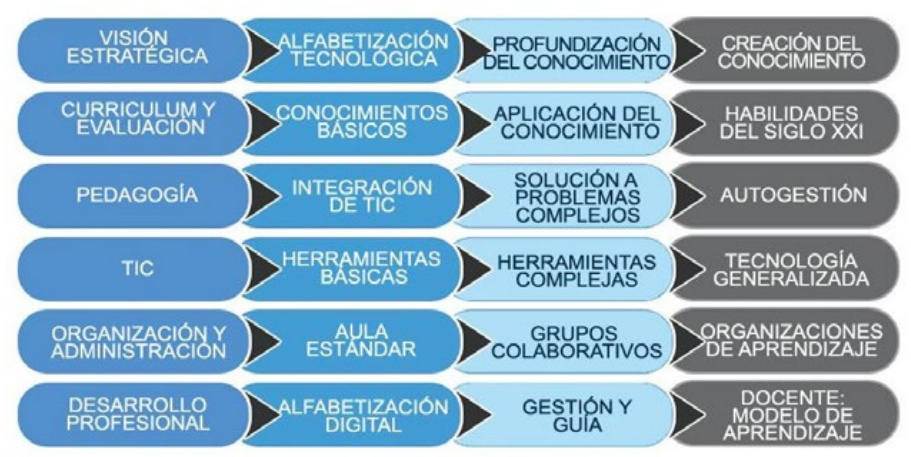

Fuente: UNESCO (2011). Adaptación.

En respuesta a las necesidades cada vez mayores en el campo educativo y a las sugerencias de la UNESCO, algunas instituciones han invertido horas de trabajo para proponer manuales, instructivos o programas que apoyen la formación del tutor a distancia. En México existe un Manual del docente de Educación a Distancia (Hernández y Legorreta, 2011) que enfoca la atención hacia el papel y funciones académico-administrativas del docente en una plataforma institucional. También está el documento titulado La tutoría en el Sistema de Universidad Abierta y Educación a Distancia de la Universidad Nacional Autónoma de México (UNAM, 2013); en este se asume la tutoría como un proceso de acompañamiento e intervención educativa individual o grupal, realizada a distancia por académicos o estudiantes de niveles superiores habituados al uso de tecnologías de la información y la comunicación; la meta es promover la retención escolar y el éxito académico de cada estudiante, a partir del diseño e implementación de acciones y estrategias personalizadas, para lograr la formación integral. El Instituto Politécnico Nacional, otra importante institución mexicana, utiliza el documento Programa institucional de tutorías (para las modalidades educativas no escolarizada y mixta) en el cual indica que el asesor y el tutor son las figuras encargadas de acompañar y facilitar la apropiación del conocimiento a través de un diálogo pedagógico, que fomenta una interlocución con los alumnos para la interpretación, reelaboración, construcción y aplicación de conocimientos y no sólo para la reproducción y memorización de los mismos (IPN, 2013). Similar orientación presenta el documento de la costarricense por Maya (1993) Educación

\section{a distancia y función tutorial.}

Diversos estudios promueven la atención hacia el adecuado funcionamiento del tutor a distancia. Abarca (2014) exhorta al tutor a ser un motivador no solo en los aspectos didácticos sino también en los tecnológicos. Lasso, Munévar, \& Rivera (2014) y Bravo (2014) proponen redimensionar la importancia de la acción tutorial en la virtualidad, viéndola desde una perspectiva tecno-pedagógica; analizan, cuestionan y proponen los conocimientos, actitudes, habilidades y/o competencias que requiere la figura del tutor en el campo de los estudios de posgrado. Freixas y Ramas (2014) enfatizan la orientación y acompañamiento del tutor a distancia y proponen un modelo para su funcionamiento. De acuerdo con estos últimos, el papel del tutor puede ser uno de los múltiples factores de abandono en los programas a distancia, toda vez que se puede presentar en el estudiante una sensación de soledad cuando el acompańante (tutor) no responde como guía o apoyo para resolver sus dudas.

Lo anterior sirve de base para concebir al tutor a distancia como el agente, conocedor de un tema o contenido, que promueve el aprendizaje de los estudiantes a través de la orientación y realimentación ejercida mediante una diversidad de recursos comunicacionales. Esta persona debe reunir una diversidad de atributos que le permitan establecer la cordialidad afectivo- social con sus tutorados, a quienes debe ayudar a sortear los obstáculos que se presenten en el contexto tecnológico y en lo humano. Lo anterior implica que el tutor tenga la afabilidad suficiente para mantener motivados a los alumnos de esta modalidad, que sea claro en su discurso, cuente con elementos pertinentes para que el estudiante logre los objetivos educacionales del programa bajo su responsabilidad y se adapte a las características del grupo de alumnos y a las de cada individuo.

Si bien lo expuesto en párrafos anteriores parece ser indiscutible en términos de las cualidades o características que debe poseer un tutor a distancia, sea que se incorpore de manera incidental -sin preparación especializada- o por medio de cursos, talleres o diplomados, el punto a tratar en este artículo son las posibles implicaciones que 
derivan de un incumplimiento de dichas características.

Se pueden suponer muchos efectos como consecuencia de no contar con tutores a distancia que respondan a las expectativas mencionadas. Las suposiciones pueden rechazarse fácilmente, sin un sustento. El sustento para rechazarlas debe tener base científica, solo así se pueden sostener las tesis que de ello emanen.

\section{Las orientaciones del discurso en las interac- ciones tutor-estudiante}

En toda situación educativa prevalece y se produce el acto comunicativo. Esta es una experiencia de intercambio y construcción conjunta entre docentes, estudiantes y contenidos. En el campo de la Educación a Distancia, este proceso se fortalece con el apoyo de las Tecnologías de la Información y la Comunicación, en las cuales el intercambio se realiza en los llamados Entornos Virtuales de Aprendizaje (Avila y Bosco, 2001). El discurso escrito asume un papel fundamental; dependiendo del diseño pedagógico, los intercambios de discurso se ejercen en foros, chats, mensajes instantáneos y otras variantes que ofrecen los recursos Web, como las plataformas educativas (Hernández , 2001). Así, tutores y estudiantes realimentan su aprendizaje por medio del lenguaje escrito. Frente a esta situación, el análisis del discurso puede aportar información importante acerca de la interrelación entre los actores del proceso $(\mathrm{Ca}-$ ballero, 2012; Cabero, 2004; Machado \& Campos, 2013).

Respecto al proceso de realimentación orientada al aprendizaje, el tema ha sido tratado desde los albores de la psicología educativa cuando Skinner explicó la importancia del feedback para fortalecer lo aprendido (Holland y Skinner, 1980). El tema fue rescatado, desde la perspectiva cognoscitiva, por David Ausubel y sus seguidores (Ausubel, 1968 y 1978). En el contexto de la Educación a Distancia, vale la pena destacar la síntesis que Carlos Roman hiciera de las ideas de varios autores y estudiosos; consideró que el acto comunicativo es "...el retorno de información sobre el resultado de una actividad o un proceso...", "(un proceso)... relacionado con la información que el maestro proporciona al estudiante sobre el logro de las metas de formación propuestas (Román, 2009).

De acuerdo con Díaz, Toledo y Segura (2013), así como Freixas y Ramas (2014), el tutor a distancia debe valorar la importancia de su papel como elemento motivador y guía para el estudiante; ello se materializa claramente mediante la interacción escrita con sus tutorados. De ahí que uno de los retos del tutor es realizar un análisis del discurso propio o del estudiante para identificar puntos de atención que coadyuven al proceso educativo. Con el análisis del discurso se puede identificar, desde un enfoque lingüístico, la estructuración de los intercambios; desde un abordaje cognitivo se puede examinar la construcción de significados y, desde un enfoque socio pedagógico, se puede identificar el estilo o formas de interacción de los tutores y de los estudiantes (Morán, 2008). Con estos tres referentes contextuales, existe la posibilidad de realizar un análisis desde la perspectiva psicoeducativa.

De esta última perspectiva es interesante focalizar la atención hacia las expresiones del tutor al realimentar las actividades de aprendizaje de sus estudiantes y, en su caso, los estilos -las formas- que utiliza en su discurso para motivarles. El análisis del discurso de los tutores en la educación intenta dar con la intención del sujeto parlante más allá de los propios enunciados, debido a que se trata de rehacer otro discurso, recobrar las palabras y los manifiestos de los contenidos de la interacción desde una aproximación lingüística (Turizo, 2014). O, como aseveró Van Dijk (1999), se trata de una lingüística crítica que puede encontrarse en áreas tan diversas como la pragmática, el análisis de la conversación, el análisis narrativo, la retórica, la estilística, la sociolingüística interaccional, la etnografía o el análisis de los media, entre otras. Entonces, no está sustentada en un marco teórico, más bien es una aproximación metodológica. En este tema coincide Manzano (2005), cuyos aportes metodológicos se rescataron en esta investigación.

El análisis del discurso se deriva de una transdisciplina de las ciencias humanas y sociales que estudia sistemáticamente el discurso escrito y hablado 
Análisis de las orientaciones de los estilos de interacción del tutor a distancia con sus estudiantes: aportes para mejorar la eficacia del proceso tutorial

como una forma del uso de la lengua, en tanto evento de comunicación y de interacción, en sus contextos cognitivo, social, político, histórico y cultural. Como afirma Pedro Santander, no existe una técnica específica para realizar un análisis del discurso. Aunque Manzano (op.cit.) propone realizar una clasificación y categorización del discurso apoyándose en una lógica derivada del interés que tenga el investigador. Manzano, asume gran parte de las recomendaciones de Santander (2011), similares a las de Jorge Ruíz (2009) y los aportes de Carmen Varguillas (2006) en el campo pedagógico. En apoyo a la propuesta de la referida estrategia para analizar el discurso de las interacciones entre tutor y estudiante, se pueden establecer clases de discurso basándose en las palabras y sus contenidos. El trabajo que antes se realizaba manualmente para el análisis cualitativo hoy en día es más fácil con instrumentos dedicados especialmente a esa tarea. En esta investigación se eligió "OpenCode", recurso que ofrece en línea la Universidad de Umea; esta herramienta facilita el reporte cuantitativo de los elementos o códigos que deriven del análisis cualitativo.

\section{Una universidad en línea con problemas}

Se presenta el caso de una Universidad a distancia, institución de educación superior privada dedicada íntegramente a la formación de profesionales en diversas áreas del conocimiento, con planes de estudio a distancia. Esta institución cuenta con Escuelas de Humanidades, Tecnología, Negocios y Ciencias de la Salud. Todos los estudiantes son atendidos por medio de un tutor a distancia. La institución ostenta un modelo educativo centrado en el estudiante, quien interactúa en un ambiente colaborativo y, con apoyo, tutorial logra su formación y desarrollo buscado. Dicho ambiente pretende incentivar la colaboración entre los alumnos para conocer, compartir y ampliar el conocimiento de cada uno de ellos.

De acuerdo con los postulados institucionales, la tutoría especializada está dedicada a brindar acompañamiento a los alumnos, apoyándolos en sus necesidades con la finalidad de facilitar su inserción en el ámbito escolar y laboral, así como fomentar el ambiente colaborativo. Su plataforma para soportar el trabajo tutorial está disponible las 24 horas de los 365 días del año.

Un grupo de expertos, el Consejo Académico, conformado por profesionales reconocidos en los diferentes ámbitos profesionales y académicos, valida sus programas educativos. Ante dicho Consejo se reportaron algunas complicaciones con los estudiantes, además de comentarios relacionados con la insatisfacción de los estudiantes por la forma de trabajo, los coordinadores de los programas de carrera coincidieron en la identificación de deserciones relativas (no definitivas) de un porcentaje aproximado, mayor al $20 \%$ de los estudiantes en los últimos periodos semestrales. Dicha situación cobró gran interés, ya que se había efectuado la validación académica por parte del Consejo y con las primeras generaciones de estudiantes no se había presentado un problema similar. Entre otras suposiciones acerca de los orígenes del caso, se consideró que el papel del tutor tenía algo que ver, por lo que se propuso prestar más atención a este.

Las diversas sugerencias motivaron que una comisión formulase una propuesta basada en la investigación aplicada, con la cual se esperaba responder a las preguntas: ¿Son los tutores quienes influyen en la deserción o insatisfacción de los estudiantes? En su caso, ¿Qué factores influyen en la eficiencia de la función del tutor? En el intento por responder a estar y otras preguntas colaterales se planteó la siguiente hipótesis:

H0. Los inadecuados estilos de interacción de los tutores a distancia motivan la deserción de los estudiantes de la Universidad. La investigación para probar esta hipótesis tuvo como objetivo identificar los estilos de interacción de los tutores para realimentar y motivar a los estudiantes.

Cabe señalar que el presente estudio reporta la primera parte del proyecto exploratorio, mientras las autoridades de la Universidad a distancia entregan los datos que permitan evaluar el desaliento mencionado.

Por lo anterior es que, para esta fase del estudio en desarrollo, se contempla la siguiente hipótesis:

H0. Los estilos de interacción de los tutores a distancia se orientan hacia la realimentación del 
aprendizaje del estudiante de la Universidad a distancia.

Desde ya, ha de quedar claro que este estudio responde a los principios de la investigación aplicada, toda vez que intenta resolver un problema específico. Si bien puede ser aprovechado el tema y el método aquí expuesto, cada caso tendrá características propias que deben tomarse en cuenta.

\section{MATERIALES Y MÉTOdo}

\section{Tipo y diseño}

Se trata de una investigación exploratoria comparativa, de corte longitudinal, basada en el análisis del discurso en interacciones tutor-estudiante, de diversas fases.

\section{Población}

El estudio estuvo compuesto por una población de 46 tutores. Para la selección de la muestra se aplicó un muestreo no probabilístico, discrecional (Larios y Figueroa, 2008), así mismo se consideró como criterios de exclusión a los tutores de ingreso reciente al sistema y no activos, siendo 10 los excluidos, quedando la muestra con 36 tutores considerados como activos, realizándose en base a las 6865 interacciones tutor-estudiante registradas en la plataforma durante los dos últimos periodos semestrales (10 meses) por ellos; de forma tal que se obtuvo el promedio de dichas interacciones por tutor y se separó a cinco de ellos, los que rebasaban una desviación estándar $(\sigma=123)$ superior a la media (148).

Asimismo considerando la ética y confidencialidad de los participantes tutores se ha omitido el nombre de la institución educativa y el nombre de ellos, quienes son profesionales especializados en diversas disciplinas, cuentan con un mínimo de estudios a nivel de licenciatura y experiencia docente mínima de un año. Asignándose a cada tutor un numeral. Solo la institución educativa sabe a quién corresponde dicho numeral.

El instrumento para la compilación de los datos fue la propia plataforma educativa que registra toda interacción del tutor con el estudiante en una base de datos, soportada en MySQL. Debido a que los datos compilados se ciñen a un esquema informático de registro automatizado, no se requiere proceso de validación ni confiabilidad.

Las variables a considerar en este estudio son:

Independiente: El tutor. Persona conocedora de un tema o contenido que promueve el aprendizaje de los estudiantes a través de la orientación y realimentación ejercida por diversos recursos comunicacionales.

Dependiente: Tipo de discurso. Estilo en el que el tutor se expresa, en interacción con el estudiante, en pro del aprendizaje de este, haciendo énfasis en el proceso de realimentación. Es la institución que contrata al tutor para tal fin quien determina su función y asignación en la muestra poblacional. La medición de las diferentes formas del discurso se hace a través del lenguaje escrito, manifestado en los chats o foros que se registran en una plataforma educativa. De manera específica se intentó identificar la realimentación orientada hacia el aprendizaje.

\section{Procedimiento.}

El trabajo se realizó en dos fases:

Primera fase. Con base a un análisis de los 6,865 registros captados, se eliminaron aquellos que contenían texto insignificante para los fines del estudio. Por ejemplo: puntos suspensivos, "hola" o “smiles" (:),:).

Con esta fase de exploración se dio la propuesta inicial de las categorías siguientes.

- Socialización inicial. Palabras alusivas a salutaciones.

- ACT.: Actitud motivante. Aseveración del tutor que exhorta hacia la superación o logro personal del estudiante.

- Realimentación. Expresión del tutor que invita a mejorar la ejecución manifiesta, evitar las acciones consideradas como inadecuadas para conseguir los resultados previstos. 
Análisis de las orientaciones de los estilos de interacción del tutor a distancia con sus estudiantes: aportes para mejorar la eficacia del proceso tutorial

Segunda fase. En un primer momento se realizó el análisis del discurso. Para la categoría de realimentación se dieron de alta las siguientes clases de interacción, basadas en el discurso del tutor (las clases y códigos que aparentemente ostentaban diversas formas de interacción) hacia el estudiante y la frecuencia aparente de expresiones similares:

- CONFe.: Confusión en la entrega. Cuando se refería el tutor al espacio donde se debería subir un reporte o a la fecha de entrega del mismo.

- BIBL.: Declaración de bibliografía. Cuando el estudiante no expresaba las citas bibliográficas en su reporte, de acuerdo al formato solicitado.

- FORM.: Formato. Comentarios relacionados con el formato del documento.

- ORT.: Ortografía. Observaciones referidas a fallas en la ortografía, gramática o puntuación.

- PLAG.: Plagio. Explicaciones acerca de la identificación de plagio de documentos, sea tomadas literalmente de fuentes externas o por la reproducción parcial de contenidos vertidos en reportes entregados por los compañeros.

- INC.: Incumplimiento. Comentario acerca de la no entrega de la actividad solicitada, de acuerdo al calendario de actividades.

- INST.: Instrucciones. Argumento que indica incidencia negativa hacia las indicaciones específicas manifestadas por el tutor para realizar la actividad.

- RED.: Redacción. Cuando el estudiante presentó fallas en la representación escrita de su documento.

- FALp.: Falta de profundidad. Comentarios alusivos a la atención superficial del tema asignado y su reporte.

- CONFc.: Confusión. Cuando se explicó la falta de comprensión del estudiante hacia la actividad de apren- dizaje, ya que algunos reportes deberían expresar contenidos declarativos y otros, contenidos procedimentales.

- CARe.: Carencia de elementos. Expresiones orientadas hacia la mejora del contenido del reporte por omitir elementos solicitados en la actividad de aprendizaje.

En un segundo momento de análisis de los discursos se eliminaron los registros de la categoría Socialización, quedando 6773 registros. Las expresiones de texto que se calificaron en las categorías mencionadas se incorporaron al software para producir un reporte cuantitativo. En los casos en los cuales se presentó una aparente inconsistencia en la contabilidad, los discursos se revisaron nuevamente para calificarlos de nuevo, hasta depurarlos.

Teniendo como base el método de muestreo elegido ( $\mathrm{n}=$ registros $>1 \sigma$ de la media) se realizó el análisis del contenido de las interacciones de los cinco tutores seleccionados, omitiéndose los discursos de los estudiantes. En esta fase se analizaron en total 2081 registros.

Como se puede apreciar, con el programa informático OpenCode se rescataron las categorías y sus clases (codes).

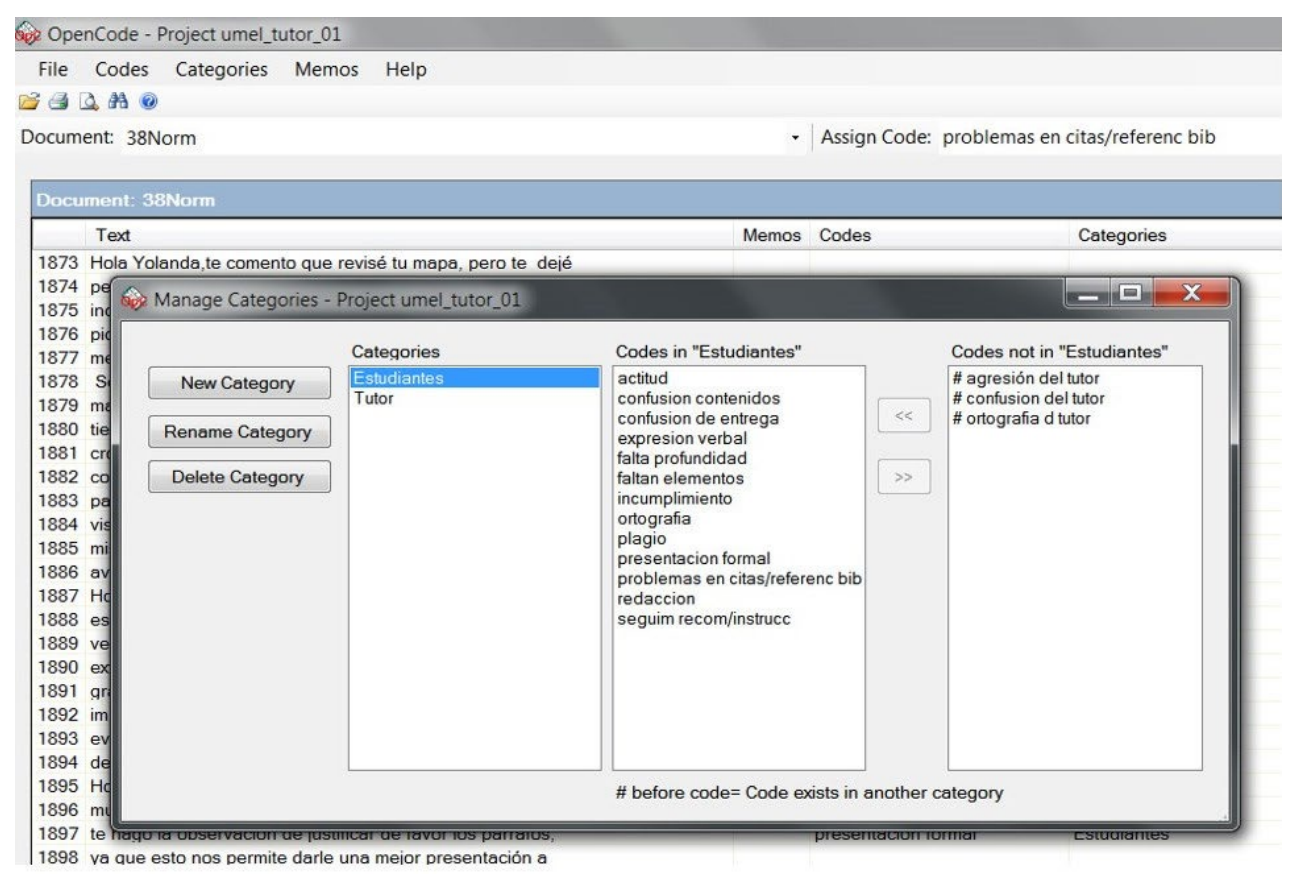

Figura 2. Ejemplo de asignación de clases y categorías de discurso en OpenCode. 
Para validar la clasificación de los discursos, se eligió una muestra aleatoria de 104 registros $(5 \%$ del total). Se solicitó a un investigador relacionado con la Educación Abierta y a Distancia que, basándose en la definición y tipo de categorías establecidas, calificara los contenidos del discurso. Sus aportes se compararon con la calificación realizada originalmente, obteniéndose una coincidencia del 78\%.

\section{Resultados}

En primera instancia es importante comentar que, de los primeros 6,673 registros de los discursos compilados, $566(8,48 \%)$ reflejan el incumplimiento de los tutorados en la remisión de los reportes de sus actividades. Ejemplos de los mensajes categorizados en este corte:

- Falta tu actividad

- Falta subir actividad

- Falta actividad

De los 2081 registros de discurso analizados 997 (48\%) referían contenidos relativamente cortos, orientados hacia la realimentación positiva por las actividades de aprendizaje o entrega de reportes de los estudiantes. Ejemplo de ello :

Hola Mir te comento que está bastante completa tu actividad sobre las distribuciones de probabilidad.

Isael: Excelente!!!! trabajo y más a la conclusión a la que llegas. GRACIAS!!!!!

\section{Felicidades son muy buenos tus trabajos!}

En los otros 1084 registros (52\%) se encontró que el $0,6 \%$ contenían expresiones con intención motivante tipo ACT. (Véase Tabla 1).
Tabla 1. Frecuencia de argumentos por categoría, por tutor

\begin{tabular}{lrrrrrr} 
Clav & Sum & \multicolumn{1}{c}{ T1 } & T2 & T3 & T4 & T5 \\
ACT & 7 & 2 & 2 & & 2 & 1 \\
CONFe & 21 & 3 & 3 & 2 & 12 & 1 \\
BIBL & 37 & 11 & 6 & 4 & 4 & 12 \\
FORM & 39 & 1 & 3 & & 9 & 26 \\
ORT & 56 & 23 & 13 & 9 & 4 & 7 \\
PLAG & 56 & 12 & 17 & 11 & 12 & 4 \\
INC & 70 & 11 & 13 & 2 & 34 & 10 \\
INST & 102 & 4 & 29 & 27 & 22 & 20 \\
RED & 109 & 26 & 25 & 30 & 11 & 17 \\
FALp & 172 & 26 & 29 & 24 & 45 & 48 \\
CONFc & 193 & 27 & 22 & 51 & 49 & 44 \\
CARe & 222 & 50 & 46 & 39 & 32 & 55 \\
Total & 1084 & 289 & 396 & 342 & 595 & 459
\end{tabular}

Como ejemplo se hace la siguiente referencia:

Hola Chris. Gracias por tu análisis, tu estilo y redacción crean todo el ambiente y reflejan a la perfección tu manera de pensar. Efectivamente el hombre es capaz de adaptarse a los cambios que se le van presentando en su vida, y es esta capacidad la que nos permite inferir cuáles son los recursos cognoscitivos, sociales, prácticos, etc. del sujeto para continuar a partir de la solución de los problemas o bien detenerse en un punto y llegar a cometer suicidio o algún tipo de trastorno mental. Sigue asi, da gusto leer documentos como el tuyo. saludos

Para la categoría Confusión en la entrega (CONFe.), el porcentaje de argumentos identificados fue de $1,9 \%$. Por ejemplo:

- Te pido de favor entregues, esta actividad, la fecha de plazo fue el 4 de noviembre.

- Hola Jess: ¿Qué pasó con el análisis de la película? en el master se estableció como fecha de entrega el 8 de noviembre.

En el caso de las observaciones acerca de la bibliografía (BIBL.), los argumentos identificados ocuparon el 3,4\%. Por ejemplo:

Muy bien Ely, has recolectado información personal de Siddhartha muy importante que te permitirá realizar el análisis de su personalidad. Por otro lado, que la bibliografía electrónica no es válida, por lo que no será tomada en cuenta. Saludos. 
Análisis de las orientaciones de los estilos de interacción del tutor a distancia con sus estudiantes: aportes para mejorar la eficacia del proceso tutorial

Para observaciones de formato (FORM.), los argumentos fueron del 3,6\%; de la ortografía (ORT.) y el plagio (PLAG.), 5,2\% en cada una; en incumplimiento (INC.), 6,5\%; respecto a las instrucciones no seguidas (INST.), 9,4\%; aquellos relacionados con la redacción (RED.) fueron el 10,1\%; los asociados con falta de profundidad (FALp.), 15,9\%; los que expresaron al estudiante confusión de contenidos o procedimientos (CONFc.), 17,8\% y los que explicaban carencia de elementos solicitados (CARe.), 20,5\%. Estos valores se representan en la figura 3 .

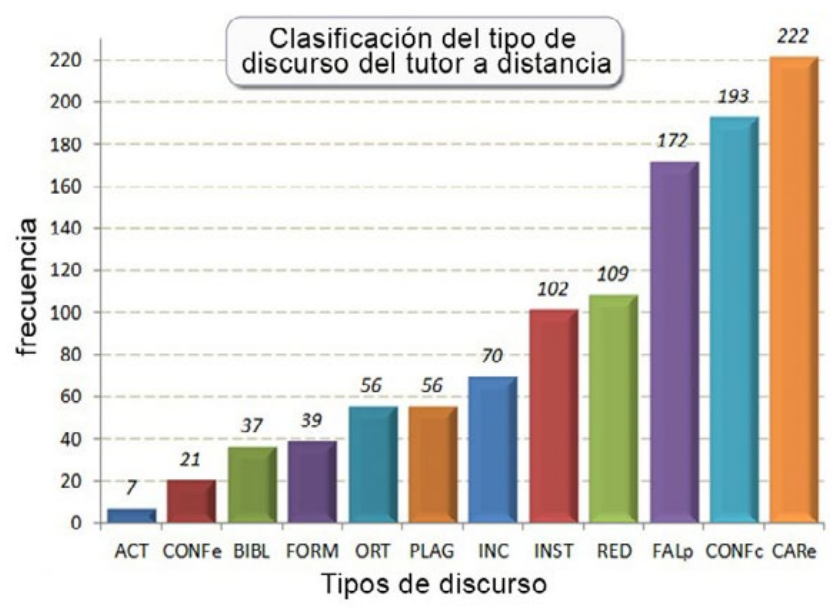

Figura 3. Frecuencia y porcentaje de discursos por categoría expuestos por los tutores.

Fuente: Base de datos de los discursos analizados.

Toda vez que la intención implícita en el estudio fue identificar el estilo de cada uno de los tutores, en la siguiente etapa se clasificaron los argumentos calificados separándolos por cada uno de los cinco tutores. En términos de frecuencia, los resultados se pueden apreciar en la siguiente tabla (se aclara que se asignó numeral a los nombres originales de los tutores para salvaguardar el anonimato).

\section{Discusión y Conclusiones}

La exposición de mensajes registrados que indican el incumplimiento en el envío de reportes de las actividades para el aprendizaje (frecuencia 566) es una clara muestra de la problemática observada. Si bien los discursos contabilizados (Falta tu actividad, Falta subir actividad y Falta actividad) representan un $8.48 \%$, existen otros con similar
Tabla 2. Frecuencia y porcentaje de discursos por categoría expuestos por los tutores ante los estudiantes.

\begin{tabular}{lrr} 
Clases de argumentos & \multicolumn{1}{l}{$f$} & $\%$ \\
ACT (Actitud motivante) & 7 & 0,6 \\
CONFe (Confusión en la entrega) & 21 & 1,9 \\
BIBL (Declaración de bibliografía) & 37 & 3,4 \\
FORM (Formato) & 39 & 3,6 \\
ORT (Ortografía) & 56 & 5,2 \\
PLAG (Plagio) & 56 & 5,2 \\
INC (Incumplimiento) & 70 & 6,5 \\
INST (Instrucciones) & 102 & 9,4 \\
RED (Redacción) & 109 & 10,1 \\
FALp (Falta de profundidad) & 172 & 15,9 \\
CONFc (Confusión) & 193 & 17,8 \\
CARe (Carencia de elementos) & 222 & 20,5 \\
\hline Total & 1084 & 100
\end{tabular}

contenido que pueden confirmar la ausencia de los estudiantes de la Universidad a distancia en los programas que se desarrollaron en el periodo de estudio.

En términos generales, los resultados sirven de base para suponer que la mayoría de los contenidos identificados en los discursos de realimentación se orientan hacia la carencia de elementos solicitados al estudiante (CARe. $=20,5 \%)$. En este punto será necesario un análisis más cuidadoso para establecer si el estudiante no comprende las consignas o estas no son lo suficientemente explícitas en las actividades de aprendizaje expuestas en la plataforma, puesto que existe una coincidencia de alta frecuencia entre todos los tutores.

De forma similar al tema de la carencia de elementos solicitados, están los de confusión acerca de los contenidos expuestos, la falta de profundidad o extensión en los reportes del alumno, así como en la declaración de la bibliografía y el formato del documento (CONFc. $=17,8 \%$, FALp. $=15,9 \%$, FORM. $=3,6 \%$ y BIBL. $=3,4 \%)$. Debido a las características de estos temas, será posible contrarrestar las faltas de precisión cuando la Universidad a distancia retome las recomendaciones que al respecto existen acerca de las rúbricas, principalmente aplicadas a la Educación Abierta y a Distancia, como por ejemplo lo expusieron Esquivias, Gasca y Martínez (2009) y recientemente Ponce y Rodríguez (2013). 
Indudablemente, el tema de la realimentación ante las fallas de redacción y ortografía del documento $(\mathrm{RED} .=10,1 \%$ y ORT. $=5,2 \%$, respectivamente) es un punto de suma importancia. Además de la expresión de los contenidos esperados en la consigna o actividad de aprendizaje, el estudiante -futuro profesional- debe poseer la cultura suficiente para expresarse por medio del discurso escrito, que debe responder a las normas propias del lenguaje. No obstante, ha de señalarse que algunos de los tutores presentan este tipo de fallas, principalmente en la ortografía. Además de una alta frecuencia de inadecuado uso de acentos y omisiones de letras, destacan errores que no deberían presentarse por parte de quien es ejemplo para el estudiante. Algunas muestras de lo mencionado se presentan a continuación:

- Te felicito, porque a pesar de que estás retomando tus estudios, has demostrado un gran compromiso y responsabilidad. ;Por favor sigue con este impetú!

- Muy vien Remy, felicidades

- En ningún momento mencionas los aspectos económicos, sociales y educativos que son las principales causas de este resago...

- ..DEBÍAS HACER ALUCIÓN AL CONTEXTO DE LOS DATOS Y TU HICISTE ENFACIS EN “COMO Y QUE”...

- no se persibio el concepto de TIC claramente, en tu presentación

- ...pués al rebizar, en ocaciones resulta incomodo hacerlo... Comentario: Se que en ocaciones no tiene tiempo... Faltó aondar un poquito más en la metodología de su aplicación...

- ...hubo un avance muy significativo en este ensayo, te felicito. Te exorto a ampliar tus conclusiones en futuros ensayos.

Como se anotó antes, esta investigación se propuso confirmar o refutar si los inadecuados estilos de interacción de los tutores a distancia motivan la deserción de los estudiantes de la Universidad.

Como una primera aproximación para descartar o aceptar la hipótesis, se identificaron los principales estilos de interacción de los tutores para realimentar y motivar a los estudiantes, encontrándose que, en la muestra estudiada, la mayoría de los discursos de los tutores orientan al estudiante acerca de la forma y extensión de los contenidos de los reportes en los que deben verter o demostrar su aprendizaje. De manera específica, se puede afirmar que la mayoría de los tutores desempeñan su función preocupándose porque el estudiante logre los objetivos del programa educativo a través de la realimentación. No obstante, será menester atender las "formas", "los estilos", los "tonos" y "las maneras" en que el tutor realiza dicha realimentación, como lo comentan Lasso et al. (2014).

Con la reserva de contar con los datos relacionados con la permanencia o abandono de los estudiantes por cada tutor, hasta este momento ya se cuenta con un referente para hacer las comparaciones respectivas con la variable "desaliento".

Antes de cerrar la presentación de este reporte, es importante señalar algunos puntos derivados de la clasificación de los argumentos en el discurso de los tutores motivaron un nuevo proceso de análisis.

En primera instancia, se encontró de manera eventual que algunos de los tutores comprendidos en la población total se expresaron de una manera que puede ser interpretada como inadecuada o impulsiva, carente de mensaje motivacional, invitación a la superación o a la mejoría de la actividad de aprendizaje. Por ejemplo:

- Dan: Qué pasó con la $2 a$ parte de tu tarea???

- ¿qué pasa?

- No entregó actividad 1 calificación "Cero “

- Ficha técnica incorrecta, planteamiento del problema incompleto, no se describen los tipos de hipótesis, tipo de análisis estadistico incorrecto.

- Buen día Alumno, no recibi correcciones de la actividad. Calificación 6...

- Alumno: hace ya una semana que se debió haber entregado ésta actividad, le pido enviarla a la brevedad, por entrega tardía se calificará ponderado a 8. Saludos.

- Jess ¿Qué pasócon tu tarea???

- Hola quedamos de que subirías la tarea el viernes pasado y hasta el momento no la he recibido. Lo que amerita que te califique con CERO. 
Análisis de las orientaciones de los estilos de interacción del tutor a distancia con sus estudiantes: aportes para mejorar la eficacia del proceso tutorial

- Hola hasta este momento no he recibido tu última tarea. Lo que amerita que te califique con Cero.

Además de la posible interpretación que los estudiantes pueden dar a estos mensajes, los tutores deben tener en cuenta que es necesario mejorar la relación socio-afectiva con el estudiante. Lo curioso de los últimos dos fragmentos tomados de los registros (numeral 8 y 9), es que fueron expresados por dos tutores diferentes. Es factible suponer que uno de ellos apoyó al otro: ¿̨usurpación de funciones?

Otro punto de interés es el hecho de haber identificado a tutores que retroalimentan a varios estudiantes exactamente con el mismo mensaje, como en los siguientes casos.

- ¡Excelente trabajo, felicidades!, no hay comentarios que realizar. (13 veces).

- ¡Muy buen trabajo, felicidades!, no hay comentarios a realizar. (19 veces).

- Buen trabajo, saludos. (52 veces).

Como se puede apreciar, estos discursos para la realimentación no declaran elementos que sirvan al estudiante para confirmar lo que han aprendido. En el último mensaje ("Falta actividad") puede provocar en el estudiante una reacción incómoda.

Por último, es necesario atender el tema de la coevaluación, pues en ninguno de los registros analizados se identificó argumento alguno en el que los tutores indicaran u orientaran a los tutorados para trabajar de manera colaborativa.

Queda entonces aplazada la última fase de este estudio en el que se intenta relacionar, en su caso, el tipo de discurso que el tutor utiliza en las interacciones con sus estudiantes y las consecuencias en la permanencia o desaliento de los mismos en el sistema de educación a distancia de esta institución educativa. Definitivamente, no es solo la función del tutor la que influye en el éxito de los estudiantes, pero su participación -cuando menos en el método asumido por la Universidad a distancia - juega un papel muy importante. Desde ya, se piensa en la posibilidad de analizar otros elementos del proceso de planeación y desarrollo de los programas que esta institución aplica en la formación de sus estudiantes.

Con los resultados de este estudio se fortalece la expectativa acerca del uso de la aproximación del análisis del discurso como estrategia metodológica que apoye la evaluación de la práctica tutorial en la Educación a Distancia.

\section{REFERENCIAS BibLIOGRÁFICAS}

Abarca, A. Y. (2014). La interacción tutor-estudiante en ámbitos de educación a distancia. Revista de Lenguas Modernas, 285-294.

Ander-Egg, E. (1997). Diccionario de pedagogía. Buenos Aires: Magisterio del Río de la Plata.

Aretio, G. L., y Ruiz, C. M. (2010). La eficacia en la educación a distancia: ‘ un problema resuelto? Teoria educativa, 141-162.

Ausubel, D. P. (1968). Educational Psychology: A Cognitive View. New York: Holt, Rinehart \& Winston.

Ausubel, D. P. (1978). In defense of advance organizers: A reply to the critics. Review of Educational Research, 48, 251-257.

Avila, M. P., y Bosco, H. M. (2001). Ambientes virtuales de aprendizaje una nueva experiencia. 20th. International Council for Open and Distance Education. Düsseldorf, Alemania.

Bates, A. W. (1999). Cultural and Ethical Issues in international Distance Education. USA: CREAD.

Bosco, H. M., \& Barrón, S. H. (2008). La Educación a Distancia en México. Narrativa de una historia silenciosa. México: Universidad Nacional Autónoma de México.

Bravo, C. J. (2014). El perfil y formación del tutor de posgrado a distancia. Memorias del 1er Congreso LAtinoamericano de Estudiantes de Posgrado en Ciencias Sociales. México: Facultad Latinoamericana de Ciencias Sociales FLACSO.

Bustos, S. A., y Coll, S. C. (2010). Los entornos virtuales como espacios de enseñanza y aprendizaje. Una perspectiva psicoeducativa para su caracterización y análisis. Revista mexicana de investigación educativa, 15(44).

Caballero, A. R. (2012). Alcances y limitaciones de la tutoría en la educación a distancia. Una mirada retrospectiva y reflexiva del Bachillerato a distancia en el Distrito Federal. (Tesis de maestría inédita). México.

Cabero, J. (2004). La función tutorial en la teleformación. En F. Martínez, \& M. P. Prendes, Nuevas Tecnologías y Educación. Madrid: Pearson Educación.

Castañeda, Y. M. (1993). Ténicas psicoeducativas y contexto de enseñanza: una aproximación cognoscitivista. Tecnología 
y Comunicación Educativas, 8(21), 42-49.

Crovi, D. D. (2009). Educación a distancia hoy: el reto de repensarse a sí misma. En C. L. Garay, Tecnologías de Información y Comunicación. Horizontes interdisciplinarios y temas de investigación (págs. 111-131). México: Universidad Pedagógica Nacional.

Díaz, M., Toledo, B., y Segura, L. (2013). Rol docente en la enseñanza virtual: prácticas y desafíos. 6to Seminario Internacional de Educación a Distancia - RUEDA, octubre 1012. Cuyo, Argentina.

Dijk, T. A. (1999). El análisis crítico del discurso. Revista Antrophos, 186, septiembre-octubre. Pp. 23-26. Barcelona, España.

Esquivias, S. M., Gasca, F. M., \& Martínez, S. M. (2009). Competencias del docente virtual universitario y a distancia: sistema de evaluación por rúbricas. En Memoria Electrónica del X Congreso Nacional de Investigación Educativa, 21-25 septiembre. Veracruz, México: Consejo Mexicano de Investigación Educativa, A.C.

Eusse, Z. O., \& Piña, G. B. (2005). El docente asesor o tutor en Educación Abierta y a Distancia. México: VirtuaIEduca 2005.

Fernández, E., Mireles, M., y Aguilar, R. (2010). La enseñanza a distancia y el rol del tutor virtual: una visión desde la Sociedad del Conocimiento. Revista Etic@net, VII(9).

Freixas, F. M., \& Ramas, A. F. (2014). Un modelo de tutoría para la educación a distancia. El caso de la UNAM. En VirtualEduca, Memorias Encuentro VirtualEduca 2014. Perú.

Gallego, R. A., y Martínez, C. E. (2003). Estilos de aprendizaje y e-learning. Hacia un mayor rendimiento académico. Revista de Educación a Distancia(7), 1-10.

García Aretio, L. (1987). Hacia una definición de Educación a Distancia. Boletín informativo de la Asociación Iberoamericana de Educación Superior a distancia, 4(18), 1-9.

García Aretio, L. (2001). La educación a distancia. De la teoría a la práctica. Barcelona, Espańa: Ariel.

García, A. L. (1999). Perspectivas sobre la función tutorial. Madrid: UNED.

García, A. L. (2012). La educación a distancia hoy. Modelos, eficacia y tendencias. Contextos Universitarios Mediados, 12(23).

García, A. L., Ruíz, C. M., y Domínguez, F. D. (2007). De la educación a distancia a la educación virtual. (Ariel, Ed.) Barcelona, españa.

Hernández, A. L., y Legorreta, C. B. (2011). Manual del docente de Educación a Distancia. (S. d. Virtual, Ed.) Pachuca, Hidalgo. México: Universidad Autónoma del Estado de Hidalgo.

Hernández, C. N. (2001). El chat como herramienta de comunicación en la educacion a distancia: usos y potencialidades para fomentar el aprendizaje cooperativo. Docencia Universitaria.

Hernández, G. M. (enero de 2011). La Tutoría en el Bachi- llerato a Distancia de la UNAM. Boletín SUAyED, 27(11).

Holland, J. G., y Skinner, B. F. (1980). Análisis de conducta: Un manual programado. México: Trillas.

IPN. (2013). Programa institucional de tutorías. Obtenido de Escuela Superior de Ingeniería Mecánica y Eléctrica: Recuperado de: http://www.esimecu.ipn.mx/Documents/ PDF/DOCUMENTO\%20RECTOR.pdf

Larios, R. N., y Figueroa, P. G. (2008). Muestreo. Obtenido de Proyecto de seguimiento a los cursos de estadística: http:// www.estadistica.mat.uson.mx/Material/elmuestreo.pdf

Lasso, C. E., Munévar, G. P., \& Rivera, P. A. (2014). La acción tutorial en la educación virtual. Perú: Virtual Educa.

Machado, D. S., y Campos, S. m. (2013). Tutoria em ead: em busca de uma prática afetiva e efetiva do aprendizado. Congreso Internacional ABED de educación a Distancia. Salvador-Bahia, Brasil.

Manzano V. (2005). Introducción al análisis del discurso. Fundamentos metodológicos de la Psicología. Universidad de Sevilla, España. Recuperado el 18 de julio del 2013, de: http://www.aloj.us.es/vmanzano/docencia/metodos/discurso.pdf

Maya, B. A. (1993). Educación a distancia y función tutorial. San José, Costa Rica: UNESCO.

Morán, L. (2008). La educación escolar en perspectiva humanística y pedagógica. Odiseo, revista electrónica de pedagogía, 5(10).

Moreno, O. T. (enero-marzo de 2003). Creación de una propuesta institucional de tutorías para la enseñanza superior: el caso de la Universidad de Quintana Roo. Revista de la Educación Superior, XXXII(125), 93-118.

Pagano, M. C. (2008). Los tutores en la educación a distancia. Un aporte teórico. (C. U.-1. UOC, Ed.) Revista de Universidad y Sociedad del Conocimiento, 2(2).

Ponce, M. A. y Rodríguez, M. N. (2013). El trabajo colaborativo a distancia y el proceso de autoevaluación y coevaluación mediante rúbricas. En Memorias del XXI Encuentro internacional de Educación a Distancia, 2-6 diciembre. Guadalajara, México: UDGVirtual.

Real Academia Española. (13 de abril de 2015). Diccionario de la lengua espańola. Recuperado de: http://lema.rae. es/drae/

Rodríguez, F. N. (2014). El tutor frente a la educación a distancia: concepciones, funciones y estrategias tutoriales. Revista de educación, cooperación y bienestar social-IEPC(3), 51-70.

Román, M. C. (2009). Sobre la retroalimentación o el feedback en la educación superior on line. Revista Virtual Universidad Católica del Norte, 26.

Ruiz, R. J. (2009). Análisis sociológico del discurso: métodos y lógicas. Forum Qualitative Social Research, 10(2).

Sander, B. (1990). Educación, administración y calidad de vida. Buenos Aires, Argentina: Santillana, Aula XXI.

Santander, P. (2011). Por qué y cómo hacer Análisis de Dis- 
Análisis de las orientaciones de los estilos de interacción del tutor a distancia con sus estudiantes: aportes para mejorar la eficacia del proceso tutorial

curso. Cinta moebio, 41, 207-224.

SEP. (17 de 08 de 2009). Norma técnica de competencia laboral NURUR005.01 Tutoría de cursos de formación en línea. México: Secretaría de Educación Pública. Recuperado de: http://www.conocer.gob.mx/pdfs/comites2/04rural/ 01vigentes/NURUR005.01.pdf

Turizo, A. M. (2014). Preponderancia del componente socio afectivo en el discurso del tutor virtual. En V. Educa, Memorias del XV Encuentro internacional Virtual Educa. Perú: Virtual Educa.

UNAM. (2013). La tutoria en en Sistema de Universidad Abierta y Educación a Distancia de la UNAM. México: Universidad Nacional Autónoma de México.

UNESCO. (2011). ICT Competency fremework for teachers. Francia: UNESCO.

Varguillas, C. (2006). El uso de atlas-Ti y la creatividad del investigador en el análisis cualitativo de contenido upel. Instituto pedagógico rural el Mácaro. Revista Laurus, 12, 73-87. 